\title{
Sedimentos carbonáticos bioclásticos do Atol das Rocas, Atlântico Sul Equatorial
}

\author{
Marcelo de Oliveira Soares ${ }^{1,2}$, Valesca Brasil Lemos ${ }^{3}$ \& Ruy Kenji Papa de Kikuchi ${ }^{4}$
}

\begin{abstract}
Resumo Atóis são recifes que tanto geram quanto acumulam sedimentos carbonáticos. Os carbonatos bioclásticos do Atol das Rocas, Nordeste do Brasil, foram analisados a partir de amostras superficiais de diversos compartimentos ambientais. Os resultados mostram a predominância dos bioclastos de algas calcárias, foraminíferos e moluscos nos sedimentos revelando a dominância destes grupos na bioconstrução do recife e na dinâmica sedimentar da área. Evidências de contribuições alóctones e autóctones na composição sedimentológica do atol e variações na composição ao longo do gradiente sotavento-barlavento são discutidas. Diferente de outros atóis do planeta, o Atol das Rocas apresenta as fácies lagunares com baixa proporção das fácies carbonáticas totais associadas ao recife e baixa frequência de bioclastos de corais escleractíneos.
\end{abstract}

Palavras-chave: sedimentologia, carbonatos, Atol das Rocas, Atlântico Sul Equatorial.

\begin{abstract}
Bioclastic carbonate sediments of the Rocas Atoll, Equatorial South Atlantic. Atolls are reefs that are both generate from as accumulate of carbonate sediments. The bioclastic carbonates of Rocas Atoll, Northeast of Brazil, were analyzed from surface samples of various environmental compartments. The results show the dominance of calcareous algae bioclasts, foraminiferans and molluscs in sediments of reef bioconstruction and the sediment dynamics of the area. Evidence of allochtonous and autochtonous contributions in the sedimentological composition of atoll and variations in the composition along the windward-leeward gradient are discussed. Unlike other atolls of the planet, the Rocas Atoll present the lagoonal facies with low proportion of total carbonate facies associated with the reef and low frequency of coral bioclasts.
\end{abstract}

Keywords: sedimentology, carbonates, Rocas Atoll, Equatorial South Atlantic.

\section{INTRODUÇ̃̃O}

Recifes e sistemas carbonáticos Um recife biogênico é, segundo Lowenstam (1950), “.... o produto da atividade construtiva e agregadora/cimentadora de constituintes bióticos que, devido ao seu potencial de resistência às ondas, erigem estruturas topográficas resistentes a elas". Para Cloud (1959), qualquer recife, a qualquer tempo, é o resultado de sua cadeia de nutrientes e do produto de sua desintegração, e da sua história geológica. Stoddart (1969) acrescenta ainda a esses fatores a história geológica. Essa construção é, portanto, o resultado da atividade orgânica em resposta aos fatores ambientais (hidrofísicos e hidroquímicos) que são incorporados ao registro geológico.

Muitos dos carbonatos de recifes biogênicos são produzidos diretamente através da intermediação de processos biológicos e bioquímicos, ou mesmo pela precipitação direta a partir da água do mar. As famosas manchas brancas, compostas por agulhas de aragonita ou "whitings", podem representar o processo de precipitação química acontecendo nos dias de hoje. A principal con- tribuição nos mares tropicais atuais provém dos organismos fototróficos (corais hermatípicos, algas vermelhas e verdes) e não fototróficos (foraminíferos, moluscos, briozoários e equinodermos) (Barry et al. 2007).

No sistema siliciclástico o preenchimento é formado por diversas contribuições exógenas, enquanto no sistema carbonático os sedimentos são majoritariamente gerados in situ, dentro da própria bacia de sedimentação pela biota. Como conseqüência da contribuição biogênica, fatores como latitude, temperatura, salinidade, profundidade da água, intensidade da luz, turbidez, circulação oceânica, pressão de $\mathrm{CO}_{2}$ e suprimento sedimentar, atuam de forma conjunta, e criam condições necessárias para a proliferação dos organismos formadores da "fábrica carbonática" (Hanford \& Loucks 1993).

Sedimentologia e atóis Plataformas carbonáticas largas, plataformas isoladas e atóis são sistemas dinâmicos que tanto geram quanto acumulam sedimentos. Tais sistemas carbonáticos são influenciados significativamente por oscilações eustáticas e por controles batimétrico e de

1 - Universidade Federal do Rio Grande do Sul, Instituto de Geociências, Departamento de Paleontologia e Estratigrafia, Porto Alegre (RS), Brasil. E-mail: bio_marcelo@yahoo.com.br

2 - Universidade Federal do Piauí, Centro de Ciências da Natureza, Departamento de Ciências Naturais e Arqueologia, Teresina (PI), Brasil.

3 - Universidade Federal do Rio Grande do Sul, Instituto de Geociências, Departamento de Paleontologia e Estratigrafia, Porto Alegre (RS), Brasil. E-mail: valesca.lemos@ufrgs.br

4 - Universidade Federal da Bahia, Instituto de Geociências, Laboratório de Estudos de Recifes de Corais e Mudanças Globais, Salvador (BA), Brasil.E-mail: kikuchi@ufba.br 
subsidência. As taxas de acumulação são primariamente relacionadas à influência do nível do mar e a subsidência regional. As plataformas rasas e os atóis são diretamente controlados pelo nível do mar. $\mathrm{O}$ depósito carbonático em atóis também é influenciado pela tectônica e pelas fácies sedimentares. Na sedimentologia de depósitos carbonáticos, teoriza-se que o nível do mar é um fator-chave na deposição, devido à sua importância na produção de carbonato e de espaço para sua acumulação (McNeill 2005). Segundo Aissaoui et al. (1990) o acúmulo de carbonatos nas águas rasas dos oceanos Pacífico e Atlântico é controlado pela variação eustática.

O Atol das Rocas está localizado no topo de uma cadeia de montanhas submarinas no Oceano Atlântico Sul, cuja base encontra-se a $4000 \mathrm{~m}$ de profundidade no leito oceânico, a $3^{\circ} 51^{\prime} \mathrm{S}$ de latitude e $33^{\circ} 49^{\prime} \mathrm{W}$ de longitude, distante $266 \mathrm{~km}$ da cidade de Natal e $150 \mathrm{~km}$, a oeste, do arquipélago de Fernando de Noronha, no Nordeste Brasileiro (Echeverría \& Pires 1997; Kikuchi \& Leão 1997). O substrato é composto, principalmente, por algas calcárias incrustantes e moluscos vermetídeos (Gherardi \& Bosence 1999). Diversos estudos sedimentológicos foram desenvolvidos neste atol (Duarte 1938, Ottman 1963, Coutinho \& Morais 1970, Tinoco 1966; 1972, Kikuchi 1994, Gherardi 1996) enfocando aspectos da granulometria, composição química, cons- tituintes bióticos e mecanismos de distribuição dos sedimentos nas zonas recifais. O presente estudo tem por objetivo: analisar os bioclastos sedimentares, em termos de composição e assinaturas tafonômicas, no único Atol do Atlântico Sul Equatorial (Atol das Rocas), tendo em vista o entendimento da dinâmica sedimentar.

\section{MATERIAIS E MÉTODOS}

Área de estudo A região equatorial do Oceano Atlântico compreende a plataforma continental e a planície abissal, do norte do Ceará ao sul de Pernambuco. Nesta área, localizam-se a Cadeia Norte do Brasil e a Cadeia de Fernando de Noronha, da qual também faz parte o Atol das Rocas (Schobbenhaus 1984).

Único atol do Atlântico Sul equatorial (Fig. 1) e primeira "Reserva Biológica Marinha" do Brasil, este recife é constituído por um anel circular formado por moluscos vermetídeos, algas calcárias, foraminíferos e corais, por um depósito de areia carbonática biogênica e uma laguna de pequenas dimensões, a NE, com área de $36 \mathrm{~km}^{2}$. A parte superior do anel recifal incluindo blocos encontrados na parte oriental do anel emergem 0,5 m durante a baixa-mar, quando surgem na área interna várias piscinas naturais. Na preamar, a superície do anel fica submersa 2 ou mais metros. A temperatura média em superfície é de $27^{\circ} \mathrm{C}$ e a salinidade de 36,7 ,

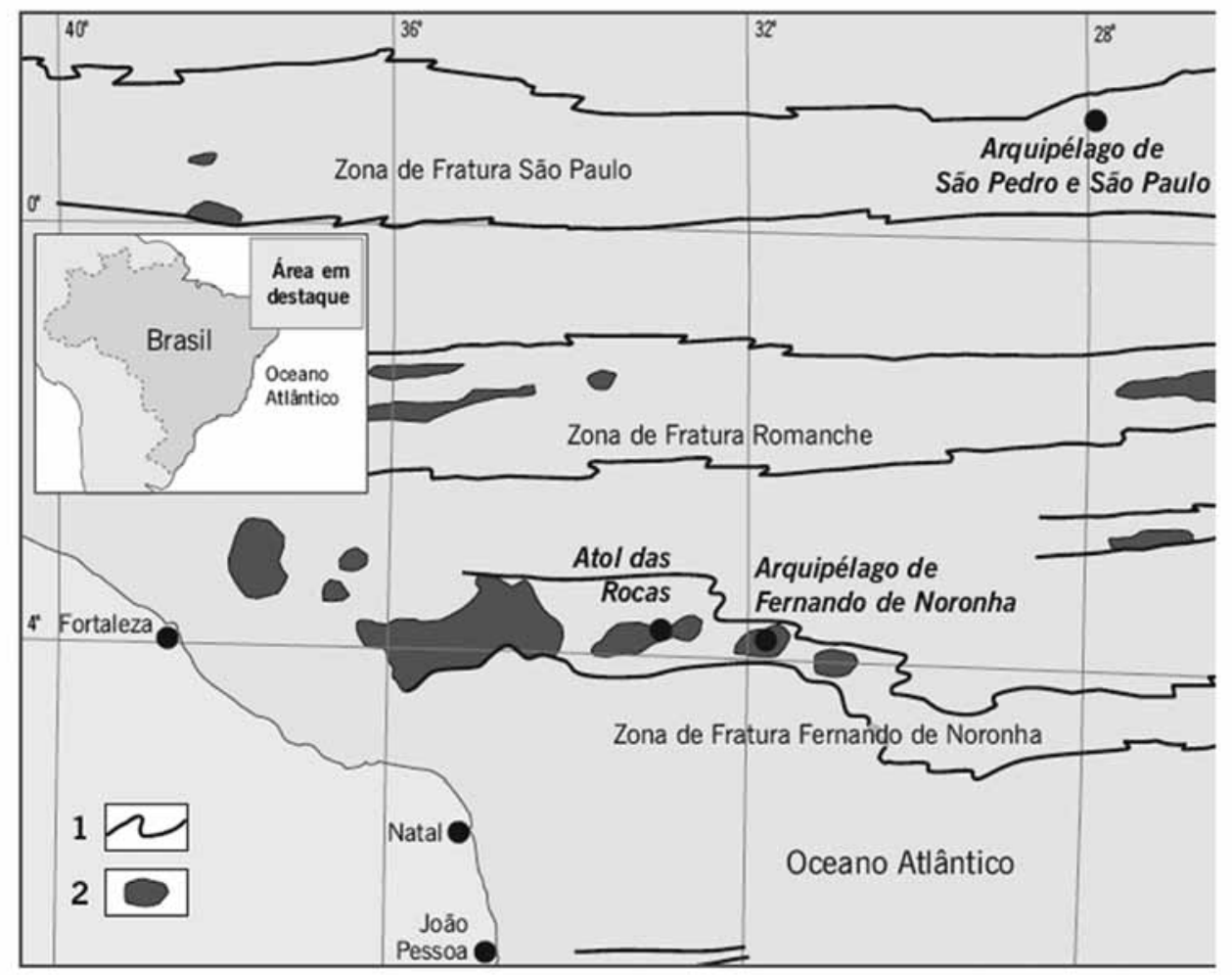

Figura 1 - Região oceânica adjacente ao Nordeste brasileiro: situação do Arquipélago de São Pedro e São Paulo na Zona de Fratura São Paulo; Atol das Rocas e Arquipélago de Fernando de Noronha, na extremidade da Zona de Fratura Fernando de Noronha. 1. Limite de zonas de fratura; 2. Rochas magmáticas. Modificado de Almeida (2006). 
tornando-se mais alta nas lagunas internas na maré baixa (Kikuchi \& Leão 1997, Gherardi \& Bosence 1999).

Coleta de campo e análises laboratoriais As coletas no recife foram efetuadas durante caminhadas no platô recifal, exposto nas marés baixas, e durante mergulhos, em apnéia e autônomo, nas partes submersas dos diversos compartimentos geoambientais. Esses dados foram completados com documentação fotográfica detalhada e com amostragens sedimentares durante o período de verão de 2008.

As amostras sedimentares foram coletadas em diversos compartimentos geoambientais do Atol das Rocas com uso de recipientes plásticos e de "core" para amostras superficiais e colocadas em sacos plásticos devidamente etiquetados. Geomorfologicamente as amostras superficiais foram obtidas da frente recifal, platô recifal, anel recifal, depósito arenoso, canais (nordeste e noroeste), ilhas (Farol e Cemitério), piscinas e da laguna na porção nordeste (Fig. 2), sendo 5 amostras para cada compartimento. O material foi acondicionado e levado ao Laboratório de Paleontologia da Universidade Federal do Ceará (UFC) e ao Laboratório de Conodontes e Foraminíferos da Universidade Federal do Rio Grande do Sul (UFRGS), para análise da constituição dos bioclastos.
Foraminíferos, coletados nas amostras sedimentares, foram fotografados ao Microscópio Eletrônico de Varredura (MEV do Centro de Microscopia Eletrônica da UFRGS) para análise de indícios de corrosão e outras possíveis feições de alteração. Os bioclastos foram colocados em stubs (pinos porta-amostras) e recobertos com uma película de ouro, para em seguida serem obtidas as imagens.

Visando atingir os objetivos propostos, foram utilizadas técnicas modernas como a estatística multivariada e a verificação das assinaturas tafonômicas para compreensão dos processos sedimentares. Para comparar a abundância dos constituintes sedimentares, foi utilizado o teste ANOVA (Análise de Variância Simples) nos diferentes compartimentos geoambientais, com pós-teste de Tukey. A análise de ANOVA buscou verificar a seguinte hipótese: As médias de abundância dos bioclastos são semelhantes em todos os compartimentos geomorfológicos? Quando se verificou uma diferença estatisticamente significante entre os compartimentos, ou seja hipótese refutada, foi utilizado o teste de Tukey para discriminar essa diferença. Tais análises estatísticas foram realizadas com nível de confiança $\alpha=5 \%$.

Análise estatística multivariada Classificação (análise

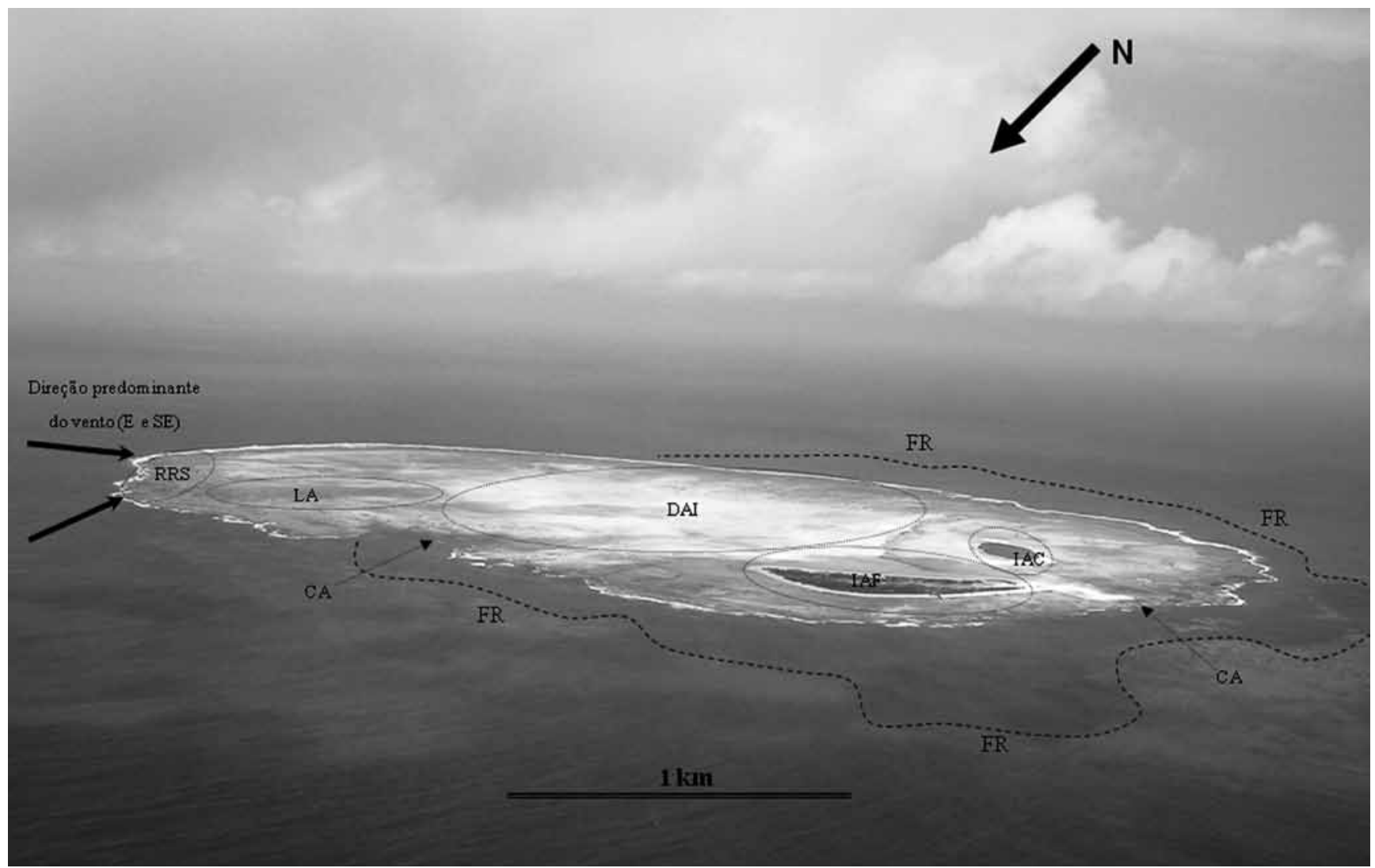

Figura 2 - Mapa geomorfológico do Atol das Rocas (Atlântico Sul Ocidental), feito a partir de aerofotografia, demonstrando a forma elipsóide, as ilhas arenosas, a sotavento, do Farol (IAF) e do cemitério (IAC). e a laguna (LA), que não ocorre na área central. Outras zonas geomorfológicas são os canais (CA), a frente recifal (FR), o depósito arenoso intermareal (DAI) e a área de resíduos de recifes (RRS). Modificado de Soares et al. (submetido). 
de agrupamento no modo Q e R) e ordenação são técnicas complementares, adotadas em uma série de áreas de estudo, como paleontologia, geologia e ecologia, para representar a estrutura dos dados e elucidar os padrões intrínsecos dentro de um conjunto de resultados (Zenetos 1991). No modo R estas técnicas agrupam utilizando as variáveis (espécies, bioclastos, etc..), enquanto no modo Q o agrupamento é baseado na similaridade das amostras.

Uma análise de dendrograma ("cluster analysis") foi utilizada para classificar as amostras sedimentares do Atol das Rocas em grupos aproximadamente homogêneos. Para esta análise, foi verificada a similaridade a partir de uma matriz de abundância dos bioclastos nos diversos compartimentos geoambientais do Atol. O coeficiente de similaridade utilizado foi o de Bray-Curtis, utilizando a estratégia de amalgamação do tipo hierárquica aglomerativa. Para corroborar os resultados da análise de cluster e obter um diagrama que permitisse a comparação com o agrupamento formado, foi realizada também a análise de escalonamento mul- tidimensional não-métrico (MDS-Multi dimensional Scaling), utilizando 999 iterações.

A análise de similaridade de Bray-Curtis foi usada com as seguintes variáveis: agrupamento das amostras sedimentares (modo-Q) e abundância e distribuição dos bioclastos (modo-R). Após este procedimento, foi construído um dendrograma utilizando o algoritmo de aglomeração do UPGMA (Unweighted Pair Group Method with Arithmetic Mean). As análises foram feitas com o uso do software estatístico Primer v. 5.0 (Plymouth Routines in Multivariate Ecological Research).

RESULTADOS O material analisado pode ser classificado como areias carbonáticas biogênicas (Fig. 3A) devido ser constituído de bioclastos de algas calcárias, moluscos, corais, foraminíferos, briozoários, equinodermos e crustáceos, com diferentes abundâncias (Tab. 1).

A maior parte dos sedimentos inconsolidados é composta de materiais bioclásticos derivados da bioconstrução recifal, como moluscos não incrustantes,
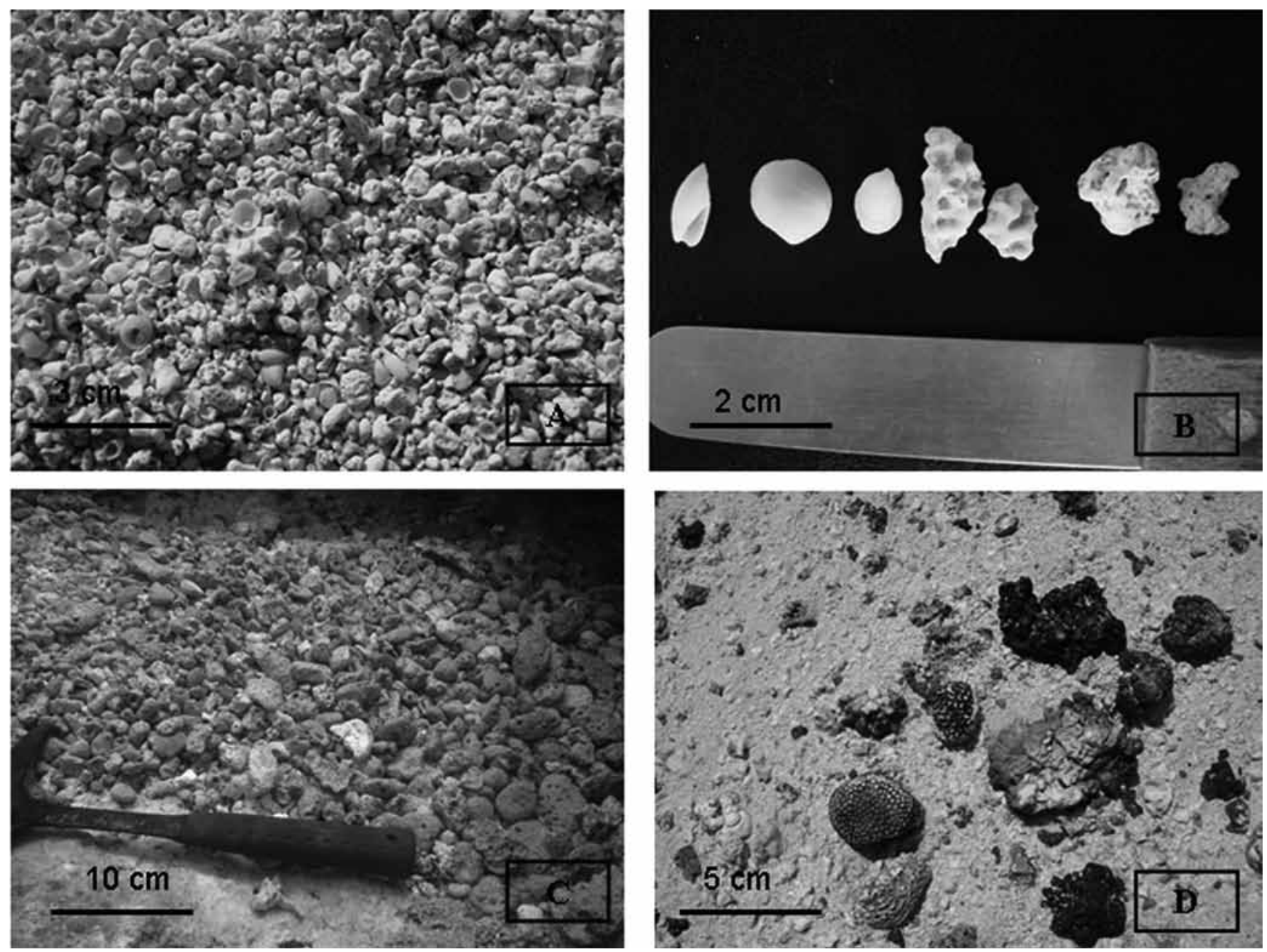

Figura 3 - Bioclastos dos sedimentos carbonáticos do Atol das Rocas. Figura 3A: Areia carbonática biogênica da Ilha do Farol. Figura 3B: Bioclastos de moluscos (três primeiros da esquerda para a direita), corais (quarto e quinto exemplares da esquerda para a direita) e de algas calcárias (sexto e sétimo exemplares da esquerda para a direita). Figura 3C: Fragmentos bioclásticos em uma piscina no lado barlavento do Atol. Figura 3D: Fragmentos de corais e algas calcárias dispostos na faixa entremarés da ilha do Cemitério. 
corais e algas calcárias (Fig. 3B). A destruição física da estrutura recifal por ação de ondas e correntes no Atol das Rocas gera várias partículas sedimentares bioclásticas (Figs. 3C e 3D). Outro importante processo de geração de sedimentos no Atol é a Bioerosão.

As algas calcárias são o componente bioclástico predominante nos sedimentos carbonáticos do Atol das Rocas (Fig. 4), revelando que tais organismos incrus- tantes e bioconstrutores geram grande quantidade de partículas através da quebra mecânica e por bioerosão.

A dominância de bioclastos de algas calcárias nos sedimentos é significante comparativamente à contribuição biogênica de outros táxons ( $\mathrm{P}<0,05$, Tukey test) (Tab. 2$)$.

Foraminíferos bentônicos vágeis possuem a mesma abundância de bioclastos de moluscos não incrustantes $(P>0,05$, Tukey test). Evidências de mate-

Tabela 1 - Taxa de ocorrência, em porcentagem, dos bioclastos constituintes dos sedimentos carbonáticos nos compartimentos ambientais do Atol das Rocas (Anel sota $=$ Anel recifal sotavento, Anel barla $=$ Anel recifal barlavento, Fren sota $=$ Frente recifal sotavento, Dep areno $=$ Depósito arenoso, Pis $=$ Piscinas, Can $=$ Canais, Ilhas = Ilhas arenosas, Lagu = Laguna, no nordeste).

\begin{tabular}{c|c|c|c|c|c|c|c|c}
\hline $\begin{array}{c}\text { Bioclastos sedimentares do Atol } \\
\text { das Rocas }\end{array}$ & $\begin{array}{c}\text { Anel } \\
\text { sota }\end{array}$ & $\begin{array}{c}\text { Anel } \\
\text { barla }\end{array}$ & $\begin{array}{c}\text { Fren } \\
\text { sota }\end{array}$ & $\begin{array}{c}\text { Dep } \\
\text { areno }\end{array}$ & Pis & Can & Ilhas & Lagu \\
\hline Algas coralinas incrustantes & 57 & 80 & 75 & 61 & 50 & 71 & 74 & 55 \\
\hline Foraminíferos bentônicos vágeis & 17 & 2 & 9 & 18 & 12 & 6 & 11 & 20 \\
\hline Moluscos não incrustantes & 13 & 9 & 8 & 13 & 16 & 12 & 10 & 9 \\
\hline Homotrema rubrum & 2 & 4 & 5 & 3 & 6 & 1 & 1 & 3 \\
\hline Corais & 1 & 3 & 1 & 1 & 1 & 3 & 2 & 3 \\
\hline Gastrópodes vermetídeos & 1 & 2 & 1 & 1 & 1 & 2 & 1 & 1 \\
\hline Millepora alcicornis & 0 & 1 & 0 & 1 & 1 & 2 & 1 & 3 \\
\hline Briozoários & 0 & 1 & 0 & 1 & 1 & 1 & 0 & 1 \\
\hline Crustáceos & 1 & 1 & 1 & 1 & 1 & 0 & 1 & 1 \\
\hline Equinodermos & 1 & 0 & 1 & 1 & 1 & 1 & 1 & 1 \\
\hline Halimeda sp. & 1 & 1 & 1 & 1 & 1 & 1 & 0 & 3 \\
\hline
\end{tabular}

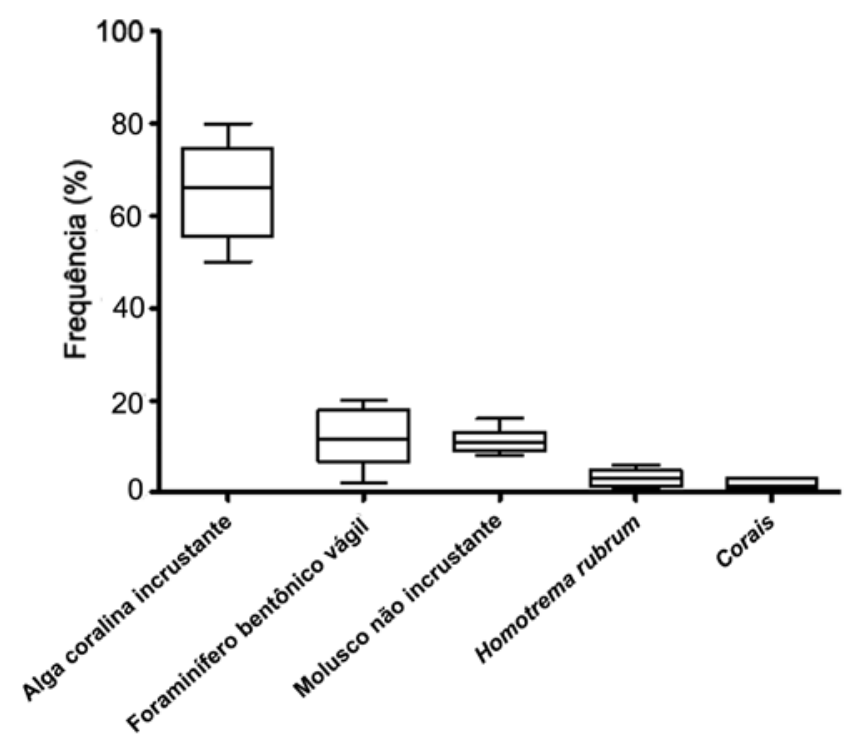

Figura 4 - Gráfico em caixa (box-whisker plot) mostrando a média e o desvio padrão da freqüência dos principais bioclastos dos sedimentos carbonáticos do Atol das Rocas. $N=40$ amostras. riais derivados da zona pelágica do oceano Atlântico Sul, como conchas de Spirula spirula, foram encontradas na ilha do Cemitério e em outros compartimentos geoambientais do Atol das Rocas (Fig. 5).

Os componentes biodetríticos predominantes são fragmentos de algas calcárias, principal organismo bioconstrutor do recife, testas de foraminíferos bentônicos vágeis e fragmentos de valvas de moluscos (Fig. 6). A análise de agrupamento no modo $\mathrm{R}$ demonstra a presença de dois grupos. Um grupo A, com baixa frequência nas zonas geomorfológicas do Atol das Rocas, formado por tafocenose constituída de restos de Millepora alcicornis, Halimeda sp., Briozoários, Vermetídeos, Homotrema, Crustáceos, Equinodermos e Corais, e um grupo B, com alta frequência e ocorrência nos diversos compartimentos geoambientais, formado por bioclastos de algas coralinas incrustantes, foraminíferos bentônicos vágeis e moluscos não incrustantes.

No dendrograma dos compartimentos geoambientais, observa-se um grupo com forte afinidade formado pelas piscinas e pelo depósito arenoso (mais de $95 \%$ de similaridade). Tal agrupamento é explicado pela presença de todos os bioclastos amostrados e por abundância semelhante (Fig. 7). Essas áreas são principalmente de importação de sedimentos carbonáticos no Atol das 
Tabela 2 - Comparações múltiplas entre os principais constituintes bioclásticos do Atol das Rocas. $* P<0,05=$ diferença significativa, $n s=$ diferença não-significativa.

\begin{tabular}{|c|c|c|c|}
\hline Comparação & $\begin{array}{l}\text { Diferença } \\
\text { da média }\end{array}$ & $q$ & $P$-valor \\
\hline $\begin{array}{l}\text { Alga coralina } X \\
\text { Foraminífero }\end{array}$ & 53.500 & 25.865 & $* \mathrm{P}<0,05$ \\
\hline $\begin{array}{l}\text { Alga coralina X } \\
\text { Molusco }\end{array}$ & 54.125 & 26.167 & $* \mathrm{P}<0,05$ \\
\hline $\begin{array}{c}\text { Alga coralina X } \\
\text { Homotrema rubrum }\end{array}$ & 62.250 & 30.095 & $* \mathrm{P}<0,05$ \\
\hline Alga coralina X Corais & 63.500 & 30.699 & $* \mathrm{P}<0,05$ \\
\hline $\begin{array}{l}\text { Foraminífero X } \\
\text { Moluscos }\end{array}$ & 0.6250 & 0.3022 & Ns \\
\hline $\begin{array}{c}\text { Foraminífero X } \\
\text { Homotrema rubrum }\end{array}$ & 8.750 & 4.230 & $* \mathrm{P}<0,05$ \\
\hline Foraminífero X Corais & 10.000 & 4.834 & $* \mathrm{P}<0,05$ \\
\hline $\begin{array}{c}\text { Moluscos X } \\
\text { Homotrema rubrum }\end{array}$ & 8.125 & 3.928 & Ns \\
\hline Moluscos X Corais & 9.375 & 4.532 & $* \mathrm{P}<0,05$ \\
\hline $\begin{array}{c}\text { Homotrema rubrum } \mathrm{X} \\
\text { Corais }\end{array}$ & 1.250 & 0.6043 & Ns \\
\hline
\end{tabular}

Rocas, não constituindo áreas-fonte do recife. Os canais (que ligam o Atol das Rocas ao Oceano Atlântico Sul) e o anel barlavento (principal área-fonte de sedimentos) estão agrupados devido à pequena quantidade de foraminíferos bentônicos vágeis e à constituição semelhante de bioclastos. A análise de MDS (Fig. 8) com baixo stress $(0,07)$ corrobora com a análise de agrupamento do dendrograma. Observa-se uma maior similaridade, proximidade dos círculos no MDS, entre as piscinas e o depósito arenoso, além dos setores a sotavento (frente e anel) que estão bem próximos na análise.

Os sedimentos do depósito arenoso intermareal, dos canais que ligam o recife ao oceano, das ilhas, dos canais de maré, da laguna e das ilhas arenosas do Atol das Rocas (Fig. 9) são compostos principalmente de materiais derivados de bioconstruções do recife biogênico.

Foram evidenciados altos níveis de energia e retrabalhamento na dinâmica sedimentar do Atol das Rocas (sentido predominantemente unidirecional E-W) através das assinaturas tafonômicas dos bioclastos sedimentares (Fig. 10). A abrasão mecânica é o principal agente de desgaste dos restos esqueléticos nas águas rasas e agitadas do atol. O grau de corrosão apresentado pelos restos bioclásticos está diretamente relacionado com o tempo de exposição na interface água / sedimento.

DISCUSSÃO O sedimento do Atol das Rocas possui constituição biodetrítica semelhante a de diversos sistemas carbonáticos recifais do Cenozóico de forma geral; entretanto, a frequência dos bioclastos apresenta diferenças em relação a outros sistemas recifais dos oceanos Atlântico e Indo-Pacífico. Na proximidade dos recifes e das ilhas do Arquipélago de Abrolhos (Nordeste do Brasil), as partículas mais freqüentes são detritos das conchas de moluscos, fragmentos de algas calcárias, corais e testas de foraminíferos (Leão 1982).

Os fragmentos da alga Halimeda sp. ou de co-

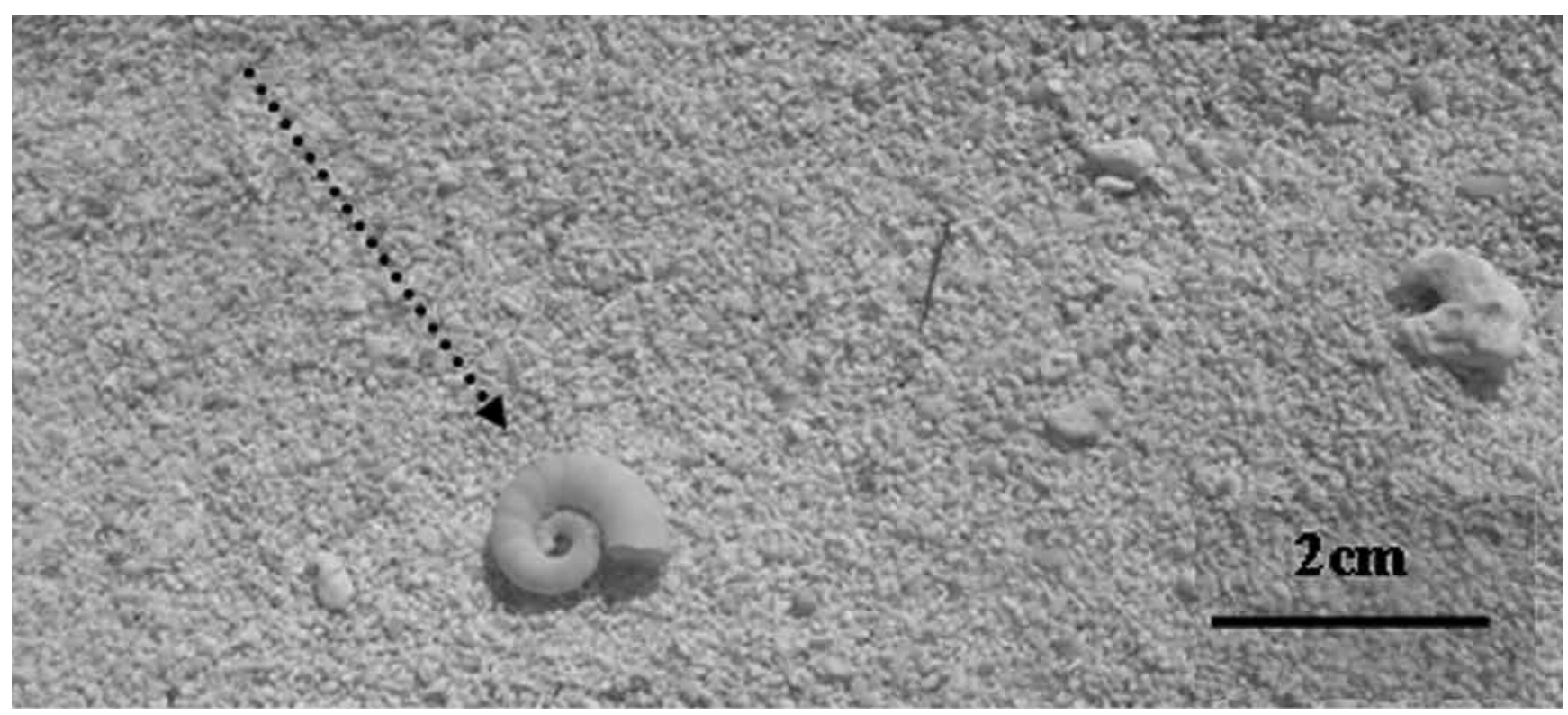

Figura 5 - Bioclasto de cefalópode Spirula spirula proveniente de zona abissal (aproximadamente $4000 \mathrm{~m}$ de profundidade) na ilha do Cemitério, Atol das Rocas, demonstrando presença na contribuição sedimentar. 
rais são os bioclastos mais comuns nos sedimentos de Rongelap e Rongerik, no Pacífico (Emery et al. 1954) Alacran (Bonet 1967), dos atóis de Bikini, Eniwetok, e no Atol de Cocos (Keeling) (Smithers 1994). Barry et

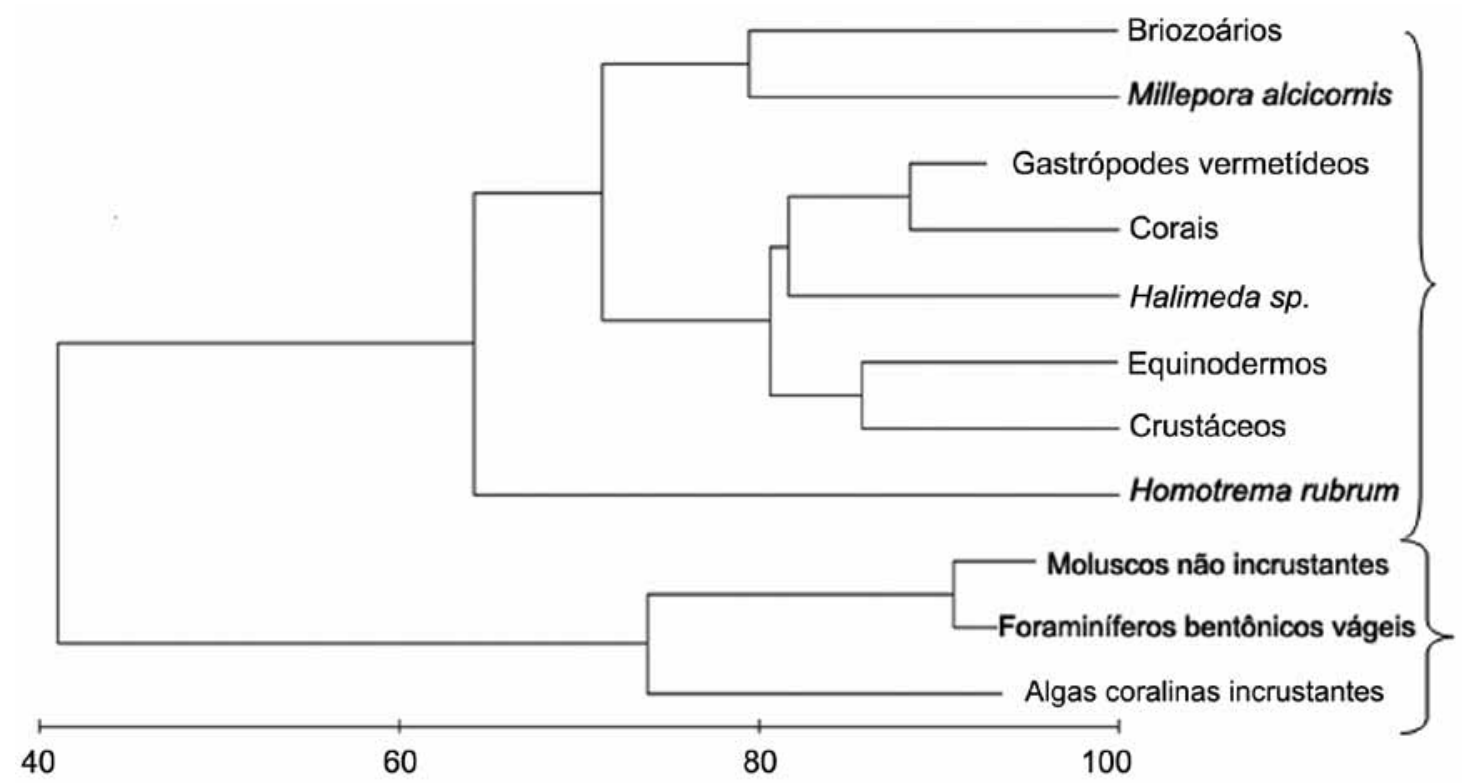

Similaridade (\%)

Figura 6 - Análise de agrupamento (cluster modo-R) dos constituintes bioclásticos do Atol das Rocas. O indice de similaridade utilizado no agrupamento é de Bray-Curtis, em porcentagem (\%). O grupo B, composto por moluscos, foraminiferos e algas, representa os bioclastos mais abundantes da tanatocenose.

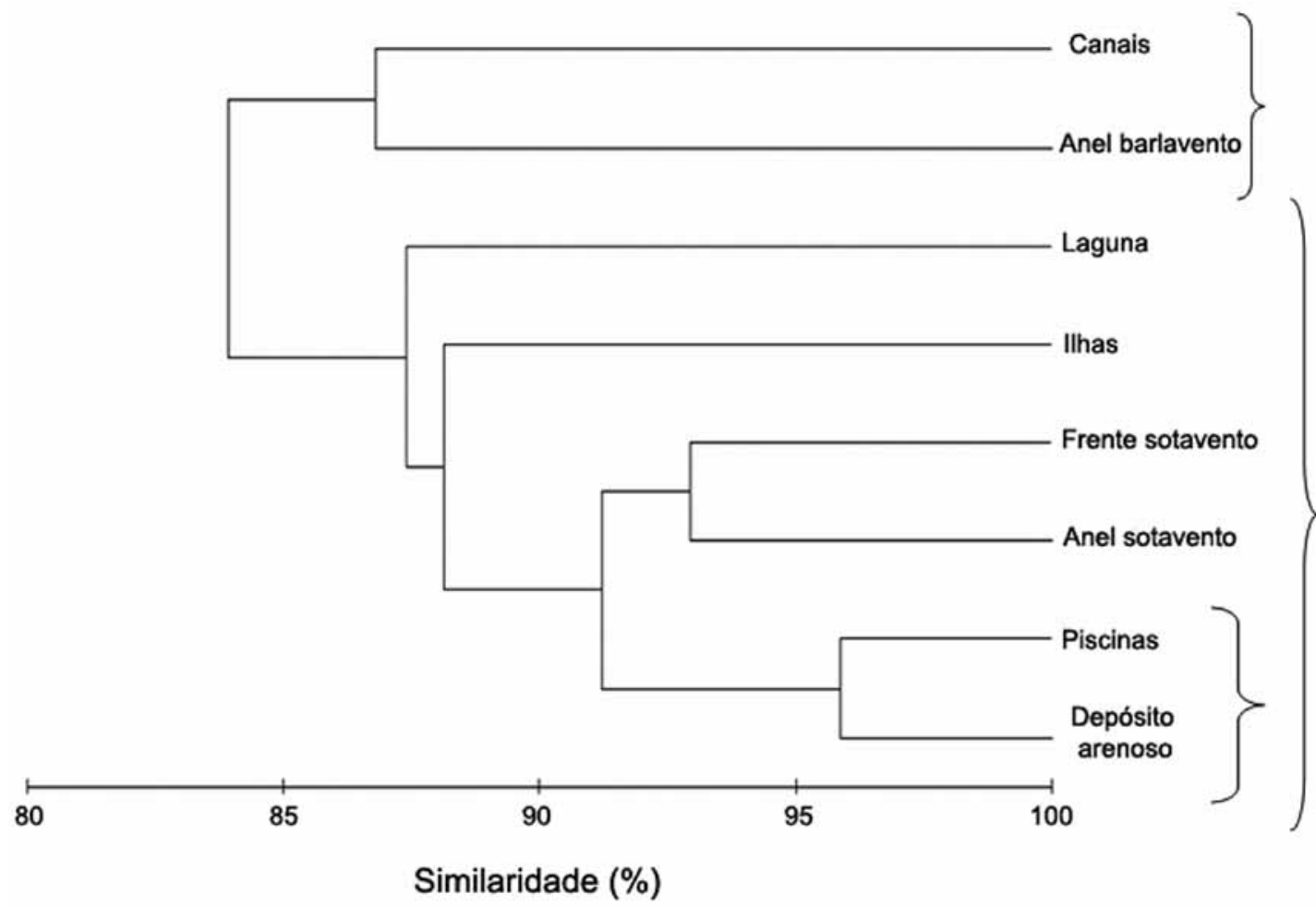

Figura 7 - Análise de agrupamento (cluster modo-Q) dos compartimentos geoambientais do Atol das Rocas. O índice de similaridade utilizado no agrupamento é de Bray-Curtis, em porcentagem (\%). 


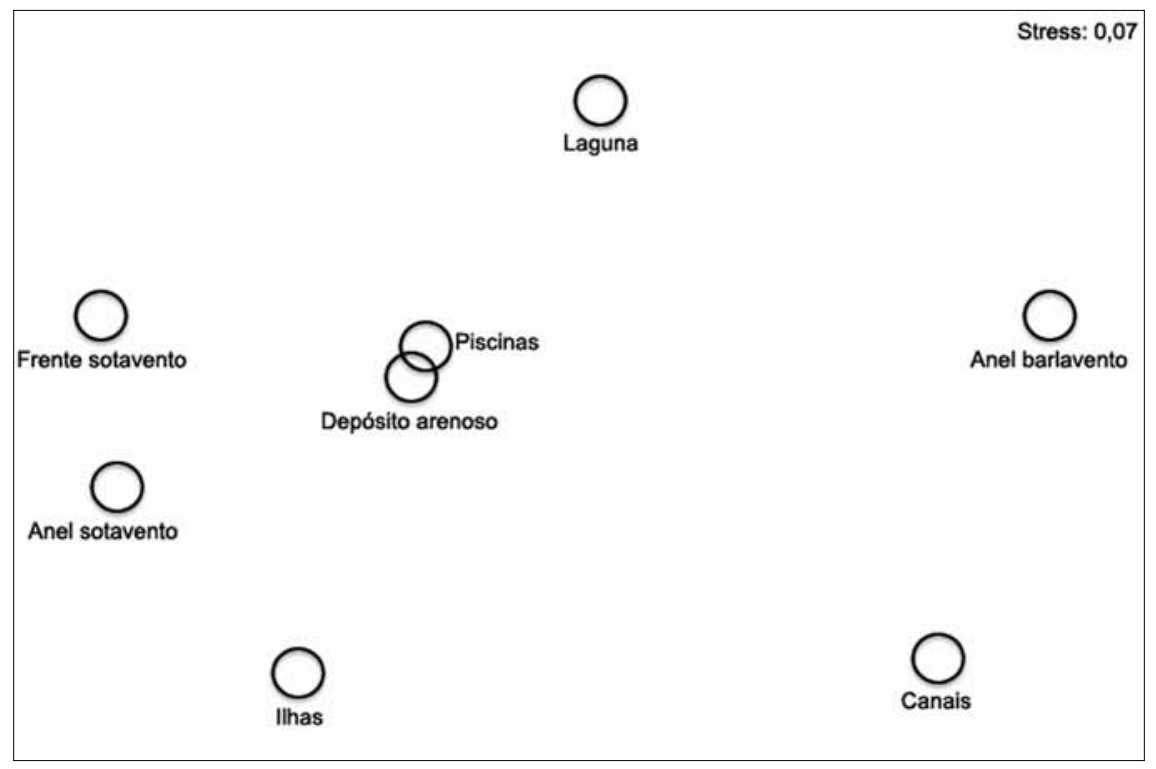

Figura 8 - Análise de MDS dos compartimentos geoambientais do Atol das Rocas. A proximidade dos círculos, que representam os compartimentos, indica maior similaridade na constituição bioclástica (táxons e abundância).
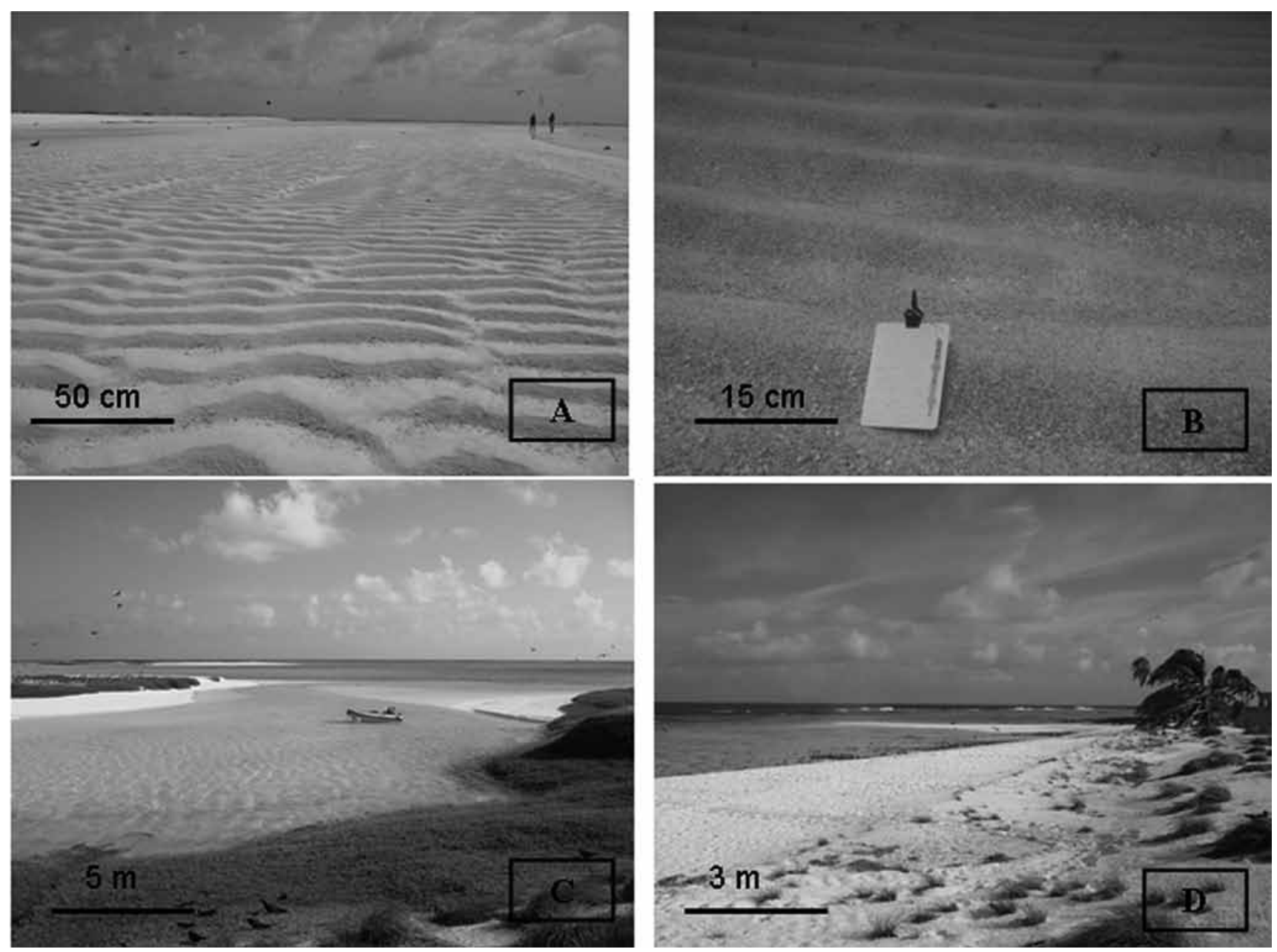

Figura 9 - Zonas de sedimentação carbonática biogênica no Atol das Rocas. (A) Depósito arenoso intermareal exibindo ôndulas em situação de maré baixa de sizígia. (B) Marcas de onda com cristas contínuas em um canal de ligação entre o Atol e o oceano adjacente. (C) Canal de maré na ilha do Farol, em periodo de maré alta. (D) Vista parcial da Ilha do Farol mostrando parte da vegetação e o acúmulo de sedimento carbonático biogênico que formou a ilha. 


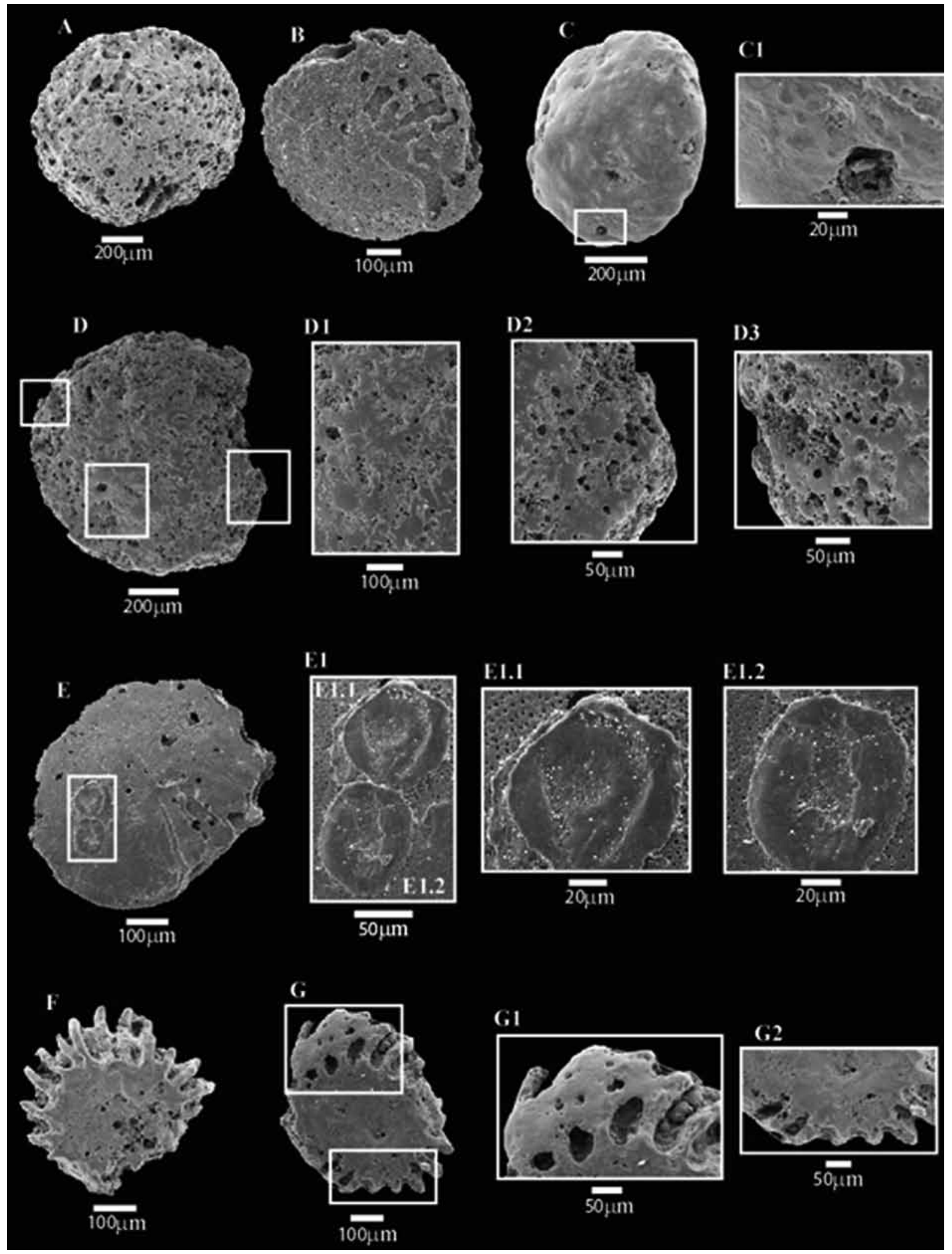

Figura 10 - Foraminiferos bentônicos e assinaturas tafonômicas indicadoras de retrabalhamento sedimentar e alta energia ambiental no Atol das Rocas analisados no MEV. A, B e C $=$ Diferentes niveis de corrosão e arredondamento. $C 1=$ Orificio na estrutura do foraminifero, evidenciando micropreenchimento bioclástico. $D=$ Altos niveis de abrasão e bioerosão no esqueleto, resultantes de retrabalhamento sedimentar e atividade geobiológica. D1, D2 e D3 representam detalhes das assinaturas tafonômicas de abrasão e bioerosão. $E$ = Incrustação calcária no esqueleto durante os processos bioestratinômicos. Detalhe de prováveis valvas (E1) incrustadas. $F$ = Foraminifero exibindo quebra e corrosão decorrente de alta energia no ambiente sedimentar do depósito arenoso entremarés. Os bioclastos sedimentares intensamente bioerodidos ou incrustados indicam, também, prolongada exposição na interface água / sedimento. 
al. (2007) em seus estudos sobre a morfodinâmica das ilhas em atóis, encontrou que os depósitos sedimentares carbonáticos têm, como constituição predominante, bioclastos derivados de organismos recifais, como corais, algas coralinas, moluscos e foraminíferos.

Duarte (1938) analisou sedimentos do Atol das Rocas e verificou apenas a constituição química. Ottman (1963), Kikuchi (1994) e Gherardi (1996) fizeram análises granulométricas e estudaram os mecanismos de distribuição dos sedimentos nos diversos compartimentos ambientais demonstrando transporte sedimentar no sentido predominante E-W (barlavento-sotavento).

Coutinho \& Morais (1970) analisaram tipologicamente as amostras sedimentares de Rocas e do Arquipélago de Fernando de Noronha, classificando-as como areias calcárias biogênicas. Os mesmos autores correlacionaram os sedimentos destas ilhas oceânicas com aqueles encontrados na plataforma continental do Nordeste do Brasil (PE ao PI), devido constituição biodetrítica semelhante. Holz \& Simões (2002) verificaram em estudos tafonômicos que o grau de corrosão apresentado por restos bioclásticos, está diretamente relacionado com o tempo de exposição na interface água/sedimento durante os eventos bioestratinômicos.

Ottman (1963) relatou que os sedimentos que formam as ilhas do Farol e Cemitério são formados predominantemente por restos de algas calcárias, como os gêneros Halimeda, Jania e Amphiroa. O conteúdo de foraminíferos foi verificado por Tinoco (1966 e 1972) e Kikuchi (1994), que constataram a dominância de $\mathrm{Am}$ phistegina sp. e Archaias angulatus.

Tsuji (1993) e Yamano et al. (2002), referindose à análise faciológica de superfície em lagunas profundas de atóis, observam que o material bioclástico é composto, principalmente, de materiais produzidos in situ em menor proporção, como algas calcárias e foraminíferos. As fácies lagunares dos atóis ocupam uma proporção significante das fácies carbonáticas totais associadas aos recifes coralíneos modernos (Yamano et al. 2002). No atol das Rocas tem-se um padrão diferenciado, pois as fácies lagunares têm pequena proporção em relação às fácies totais. Este fato é devido à pequena extensão, restrita à porção nordeste, e pequena profundidade da laguna $(<6 \mathrm{~m})$.

Identificação e classificação de fácies em atóis são comumente descritivos e só podem ser aplicados a zonas geomorfológicas específicas, como a laguna. Um esquema de classificação geral de fácies em atóis não é encontrado na literatura, diferentemente da classifica- ção de fácies em sistemas carbonáticos como plataforma continental e recifes costeiros (Tsuji 1993). Yamano et al. (2002) observam que as fácies sedimentares das lagunas dos atóis podem ser formadas de material bioclástico alóctone e material produzido in situ.

CONCLUSÕES O Atol das Rocas não apresenta corais como bioclasto dominante na composição dos sedimentos carbonáticos. A predominância na geração de sedimentos bioclásticos é de táxons de algas calcárias, moluscos e foraminíferos produzidos no recife. A presença de bioclastos, exógenos do sistema recifal, evidencia a intrusão de águas oceânicas no atol, principalmente durante períodos de tempestades, ondulações (swells) e marés de grande amplitude. A proporção das fácies da laguna, em comparação com as fácies carbonáticas totais, possui pequena contribuição devido à baixa profundidadee tamanho da laguna, restrita à porção nordeste do atol. Estas características contrastam com as de outros atóis dos oceanos Attântico e Indo-Pacífico, os quais possuem grande laguna central com profundidade de 30 a 100 m, favorável à espessa acumulação de sedimentos carbonáticos biogênicos sob baixa energia. As assinaturas tafonômicas (bioerosão, incrustação, arredondamento, abrasão,etc..) encontradas nos bioclastos sugerem condições de alta energia hidrodinâmica na parte central, distintas portanto, de outros atóis dos oceanos tropicais. Estudos futuros poderão permitir a integração de dados oceanográficos e eventos geobiológicos, como de bioerosão e tafonomia, para a compreensão dos eventos sedimentares no Atol das Rocas.

Agradecimentos $\mathrm{O}$ presente artigo faz parte da tese de doutorado do programa de Pós-Graduação em Geociências da Universidade Federal do Rio Grande do Sul (UFRGS) intitulada "Geobiologia do Atol das Rocas, Attântico Sul". M.O. Soares agradece ao CNPq (Conselho Nacional de Pesquisa e Desenvolvimento Científico), pela concessão de bolsa de Doutorado; a Petrobras e Fundação SOS Mata Atlântica, pelo financiamento da pesquisa dos trabalhos de campo; ao ICMBIO (Instituto Chico Mendes de Conservação da Biodiversidade) pela concessão de licença de pesquisa (número 12228-1); a Maurizélia Brito (coordenadora da Reserva Biológica Atol das Rocas) pelo apoio imprescindível no trabalho de campo; ao Biólogo Carlos Augusto Oliveira Meirelles pelo auxílio na coleta e fotografias subaquáticas e aos revisores anônimos que contribuíram para a melhoria significativa do manuscrito. Ruy Kenji P. Kikuchi é bolsista PQ2 do CNPq.

\section{Referências}

Aissaoui D.M., Mcneill D.F., Kirschvink J.L. 1990. Magnetostratigraphy dating of Mururoa atoll and global eustasy. Earth Planet Sci. Lett., 97:102-120.

Almeida F.F.M. 2006. Ilhas oceânicas brasileiras e suas relações com a tectônica atlântica. Terrae Didatica, 2(1): 3-18.

Barry S.J., Cowell P.J., Woodroffe C.D. 2007. A morphodinamic model of reef-island development on atolls. Sedimentary Geology., 197:47-63.

Bonet F. 1967. Biogeologia subsuperficial del arrecife Alacranes, Yucatan. Universidade Autônoma de México, Instituto de Geologia, Boletin 80, 192p.

Cloud P.E. 1959. Geology of Saipan, Mariana Islands. 4. Submarine topography and shoal-water ecology. US 
Geological Survey Professional Papers., 280:361-445.

Coutinho P.N. \& Morais J.O. 1970. Distribución de los sedimentos en la plataforma continental norte-nordeste del Brasil. In: Symposium on inventory of resources of the caribbean sea and adjoining region, 1, Atas, 284p.

Duarte P.J. 1938. O Atoll das Rocas. Recife, Arquivo de Pesquisas Agronômicas, 1:61-70.

Echeverría C.A. \& Pires D.O. 1997. Cnidarians of the Atol das Rocas. In: Int. Coral Reef Symposium, $8^{\text {th }}$, Proc., 1 , $736 \mathrm{p}$.

Emery K.O., Tracey J.I., Ladd H.S. 1954. Geology of Bikini and nearby atolls. U.S Geological Survey Professional Papers, 260:1-265.

Gherardi D.F.M. 1996. Recent carbonate sedimentation on the coralline-algal Atol das Rocas, equatorial South Atlantic, Brazil. Thesis in Geology, University of London, 353p.

Gherardi D.F.M. \& Bosence D.W.J. 1999. Modeling of the ecological succession of encrusting organisms in recent coralline-algal frameworks from Atol das Rocas, Brazil. Palaios, 14:145-158.

Hanford R.C. \& Loucks G.R. 1993. Carbonate depositional sequences and system tracts - Responses of carbonate platforms to relative sea-level changes. In: Loucks G.R. \& Sarg F.J. 1993. Carbonate sequence stratigraphy. American Association of Petroleum Geologists. AAPG Memoir, 57:3-41.

Holz M. \& Simões M.G. 2002. Elementos fundamentais de tafonomia. Editora da Universidade Federal do Rio Grande do Sul, 231p.

Kikuchi R.K.P. 1994. Geomorfologia, Estratigrafia e Sedimentologia do Atol das Rocas (REBIO-IBAMA) $R N)$. Dissertação de Mestrado, Instituto de Geociências, Universidade Federal da Bahia, 144p.

Kikuchi R.K.P. \& Leão Z.M.A.N. 1997. Rocas (Southwestern Equatorial Atlantic, Brazil): an atoll built primarily by coralline algae. In: Int. Coral Reef Symposium, $8^{\text {th }}$, Proc. $1,736 \mathrm{p}$.

Leão Z.M.A.N. 1982. Morphology, geology and developmental history of the southernmost coral reefs of Western Atlantic, Abrolhos Bank, Brazil. Ph.D. Dissertation, Rosenstiel School of Marine and
Atmosferic Science, University of Miami, 218p.

Lowenstam H.A. 1950. Niagaran reefs of the Great Lakes area. The Journal of Geology, 58:430-487.

McNeill D.F. 2005. Accumulation rates from well-dated late Neogene carbonate platforms and margins. Sedimentary Geology, 175:73-87.

Ottman F. 1963. "L'atol das Rocas" dans l'Atlantique sud tropical. Revue de Géographie Physique et de Géologie Dinamique, 2:101-107.

Schobbenhaus C. (ed.) 1984. Geologia do Brasil. Brasília, Ministério das Minas e Energia, Departamento Nacional de Produção Mineral, 501p.

Smithers S.G. 1994. Sediment facies of the Cocos (Keeling) Islands Lagoon. Atoll Research Bulletin, 407:1-34.

Soares M.O., Lemos V.B., Kikuchi R.K.P. (submetido). Aspectos biogeomorfológicos do Atol das Rocas, Atlântico Sul Equatorial. Revista Brasileira de Geociências.

Stoddart D.R. 1969. Ecology and morphology of recent coral reefs. Biological Reviews, 44:433-498.

Tinoco I.M. 1966. Foraminíferos do Atol das Rocas. Trabalhos do Instituto Oceanográfico da Universidade Federal de Pernambuco, 7:91-114.

Tinoco I.M. 1972. Foraminíferos dos bancos da costa nordestina, Atol das Rocas e Arquipélago de Fernando de Noronha. Trabalhos do Instituto Oceanográfico da Universidade Federal de Pernambuco, 13:49-60.

Tsuji Y. 1993. Tide influenced high energy environments and rodolith-associate carbonate deposition on the outer shelf and slope off the Miyako Islands, southern Ryukyu Island Arc. Japan Marine Geology, 113:255-271.

Yamano H., Kayanne H., Matsuda F., Tsuji Y. 2002. Lagoonal facies, ages, and sedimentation in three atolls in the Pacific. Marine Geology, 185:233-247.

Zenetos A. 1991. Re-evaluation of numerical classification methods for delimiting biofacies and biotopes in an estuarine environment. Lethaia, 24:13-26.

Manuscrito ID 13340

Submetido em 16 de janeiro de 2009 Aceito em 08 de outubro de 2009 